\title{
BMJ Open Motor activity across delirium motor subtypes in geriatric patients assessed using body-worn sensors: a Norwegian cross-sectional study
}

\author{
Sigurd Evensen, ${ }^{1,2}$ Alan Kevin Bourke, ${ }^{01}$ Stian Lydersen, ${ }^{3}$ Olav Sletvold, ${ }^{1,2}$ \\ Ingvild Saltvedt, ${ }^{1}$ Torgeir Bruun Wyller, ${ }^{4,5}$ Kristin Taraldsen ${ }^{1}$
}

To cite: Evensen S, Bourke AK, Lydersen S, et al. Motor activity across delirium motor subtypes in geriatric patients assessed using body-worn sensors: a Norwegian crosssectional study. BMJ Open 2019;9:e026401. doi:10.1136/ bmjopen-2018-026401

\section{- Prepublication history for} this paper is available online. To view these files, please visit the journal online (http://dx.doi org/10.1136/bmjopen-2018026401).

The material presented in this article was presented as an 8 min lecture at the European Delirium Association yearly meeting in 2017.

Received 30 August 2018 Revised 4 January 2019 Accepted 8 January 2019

Check for updates

(C) Author(s) (or their employer(s)) 2019. Re-use permitted under CC BY-NC. No commercial re-use. See rights and permissions. Published by BMJ.

For numbered affiliations see end of article.

Correspondence to

Dr Sigurd Evensen;

sigurd.evensen@ntnu.no

\section{ABSTRACT}

Objectives It remains unclear if geriatric patients with different delirium motor subtypes express different levels of motor activity. Thus, we used two accelerometer-based devices to simultaneously measure upright activity and wrist activity across delirium motor subtypes in geriatric patients.

Design Cross-sectional study.

Settings Geriatric ward in a university hospital in Norway. Participants Sixty acutely admitted patients, $\geq 75$ years, with DSM-5-delirium.

Outcome measures Upright activity measured as upright time (minutes) and sit-to-stand transitions (numbers), total wrist activity (counts) and wrist activity in a sedentary position (WAS, per cent of the sedentary time) during 24 hours ongoing Delirium Motor Subtype Scalesubtyped delirium.

Results Mean age was 86.7 years. 15 had hyperactive, 20 hypoactive, 17 mixed and 8 had no-subtype delirium. We found more upright time in the no-subtype group than in the hypoactive group ( 119.3 vs $37.8 \mathrm{~min}, p=0.042$ ), but no differences between the hyperactive, the hypoactive and the mixed groups (79.1 vs 37.8 vs $50.1 \mathrm{~min}$, all $p>0.28)$. The no-subtype group had a higher number of transitions than the hypoactive ( 54.3 vs $17.4, p=0.005$ ) and the mixed groups ( 54.3 vs $17.5, p=0.013$ ). The hyperactive group had more total wrist activity than the hypoactive group $\left(1.238 \times 10^{4}\right.$ vs $586 \times 10^{4}$ counts, $p=0.009)$. The hyperactive and the mixed groups had more WAS than the hypoactive group ( $20 \%$ vs $11 \%, p=0.032$ and $19 \%$ vs $11 \%, p=0.049$ ).

Conclusions Geriatric patients with delirium demonstrated a low level of upright activity, with no differences between the hyperactive, hypoactive and mixed groups, possibly due to poor gait function. The hyperactive and mixed groups had more WAS than the hypoactive group, indicating true differences in motor activity across delirium motor subtypes, also in geriatric patients. Wrist activity appears more suitable than an upright activity for both diagnostic purposes and activity monitoring in geriatric delirium.

\section{INTRODUCTION}

Delirium affects up to $50 \%$ of hospitalised older patients ${ }^{1}$ and is associated with

\section{Strengths and limitations of this study}

- We investigated motor activity across groups of hyperactive, hypoactive, mixed and no-subtype delirium in 60 acutely admitted geriatric patients with delirium.

- We diagnosed delirium according to the DSM-5 criteria and used the Delirium Motor Subtype Scale for motor subtyping.

- By use of accelerometer data, we evaluated motor disturbances in delirium as both upright activity, total wrist activity and wrist activity in a sedentary position.

- The major strengths of the study are the use of the Delirium Motor Subtype Scale and the simultaneous use of data from two accelerometer-based devices.

- The major limitations are the small number of patients in each group and the cross-sectional design.

increased risk of mortality, institutionalisation and dementia. ${ }^{2}$ The core symptoms of delirium are acute and/or fluctuating deficits in attention, alertness and cognition that are physiological consequences of an underlying medical condition, ${ }^{3}$ and old age, comorbidity and cognitive and physical impairment are the most important risk factors. ${ }^{4}$ Four motor subtypes of delirium have been identifiedhyperactive, hypoactive, mixed and no-subtype delirium. ${ }^{5}$ Most studies have found the highest mortality in patients with the hypoactive subtype. ${ }^{6-8}$

Previous studies on delirium motor subtypes and prognosis have used different tools for subtyping, such as the Liptzin and Levkoff schema, ${ }^{5}$ the Richmond Agitation and Sedation Scale ${ }^{6}$ and the Memorial Delirium Assessment Scale ${ }^{79}$ with the two latter tools not specifically developed for motor subtyping. The concordance between subtyping tools is low ${ }^{10}$ and it is therefore difficult to compare results from these studies ${ }^{11}$ and draw firm conclusions about the 
prognosis of the different subtypes. Through systematic improvement of previous subtyping tools, Meagher et al developed the Delirium Motor Subtype Scale (DMSS) which focuses on true motor features and no associated features like behavioural or psychiatric symptoms. ${ }^{12}$ The DMSS lists four hyperactive and seven hypoactive features. Patients with two or more hyperactive features have hyperactive delirium, and patients with two or more hypoactive features have hypoactive delirium. Patients with both hyperactive and hypoactive features have mixed delirium, and those with fewer than two motor features have no-subtype delirium. DMSS is the only validated subtyping tool, ${ }^{13}$ including validation against objective measures of motor activity. Using a thigh-worn accelerometer-based device, Godfrey and Meagher studied 25 patients with DMSS-subtyped delirium in a palliative care unit. Patients with hyperactive delirium had higher amounts of motor activity than those with hypoactive delirium, and patients with mixed delirium had amounts of motor activity between those of the hyperactive and the hypoactive groups. ${ }^{14}$

To our knowledge, this is the only study comparing motor activity across delirium motor subtypes by use of accelerometer-based devices. The thigh-worn device used by Godfrey and Meagher uses the inclination of the thigh to distinguish between standing/stepping (upright) and sitting/lying (sedentary) positions, ${ }^{15}$ and since many older patients are not able to stand and/or walk due to frailty, amputations and sequelae after stroke, this device might not capture all aspects of motor activity in geriatric patients. Still, there are reasons to believe that geriatric patients, independent of gait function, do express delirium motor disturbances. There is a need to investigate if there are differences in upright activity across DMSS-defined motor subtypes also in frail geriatric patients, furthermore to investigate if these patient groups do express delirium motor disturbances in other ways than upright activity. The aim of this study is thus to compare motor activity across DMSS-defined delirium motor subtypes in hospitalised geriatric patients, using midnight to midnight recordings of total wrist activity and wrist activity in a sedentary position (WAS), in addition to upright activity.

\section{METHODS}

\section{Design, settings and participants}

This is a cross-sectional study investigating motor activity by use of accelerometer-based devices in a limited time frame in a selected group of geriatric patients with verified, motor subtyped and ongoing delirium. The study was conducted at the geriatric ward at St. Olavs Hospital, Trondheim, Norway, between 6 May 2015 and 31 January 2017. The ward has 15 beds and is an integrated part of the medical department. The majority of patients are acutely admitted with medical conditions like infections, cardiorespiratory symptoms, cognitive symptoms or injuries after falls. ${ }^{16}$ The patients receive comprehensive geriatric assessment ${ }^{17}$ and care by an interdisciplinary team of physicians, nurses, physiotherapists and occupational therapists. The ward has only single bedrooms and is built to enhance orientation and physical activity. There is no use of physical restraints.

The inclusion criteria were age $\geq 75$ years and acute admittance to the geriatric ward. We did not exclude any patients due to diagnoses like dementia, prevalent delirium, other neuropsychiatric conditions or sensory deficits. Patients transferred from other wards were eligible for inclusion if acutely admitted to the first ward. Staff members included patients as soon as possible and always within 24 hours after admission. Only patients with complete 24 hours activity monitoring centred on the time of diagnosis of delirium were included in the final analysis.

We collected written informed consent from the individual patients or from a proxy if the patient had obvious signs of cognitive impairment. We did not include cognitively impaired patients who refused participation.

\section{Diagnosis of delirium and motor subtypes}

Two geriatricians (SE and OS) who had received supervision by an experienced delirium researcher (TBW), diagnosed delirium according to the Diagnostic and Statistical Manual of Mental Disorders (DSM)-5 criteria, ${ }^{3}$ stressing that there had to be a somatic precipitating cause. To diagnose delirium superimposed on dementia, we interviewed nurses and proxies and reviewed medical records to clarify that the present symptoms were not due to an existing dementia. We did delirium subtyping according to the DMSS and considered the motor subtype as stable during the observation period. ${ }^{13}$ We based the diagnoses on interviews with the patients, supplemented with information from proxies, nurses and chart reviews as described by Saczynski $e t a l^{18}$ and used all available information from the chosen 24 hours period of activity monitoring when deciding motor subtypes.

\section{Activity monitoring}

We asked the patients to wear two body-worn accelerometer-based devices during their hospital stay; one activPAL $(35 \times 53 \times 7 \mathrm{~mm}, 15 \mathrm{~g}$, activPAL, PAL Technologies, Glasgow, UK) attached to the midpoint of the anterior right thigh using a waterproof tape and one ActiGraph GT3X $(38 \times 37 \times 18 \mathrm{~mm}, 27 \mathrm{~g}$, ActiGraph, Pensacola, Florida, USA) attached to the right wrist using a wristband. A nurse or a physiotherapist not participating in diagnosing and subtyping of delirium attached the devices immediately after inclusion and registered the time of attachment, making sure the devices did not interfere with equipment for monitoring and intravenous lines. Ward nurses removed the devices during CT and MRI scans and showering, registering the time of removals and reattachments. If the patient removed one or both devices more than once, the staff considered that the patient did not want to wear the devices and did not reattach them. Patients wore the devices until discharge. A data scientist 
not involved in any other parts of the project (AKB), analysed the activity data when the recruitment of patients was terminated. Consequently, the assessors of DMSS (SE, OS) were blinded to the results of activity monitoring.

\section{ActivPAL outcomes}

The activPAL uses the inclination of the thigh to distinguish between upright and sedentary positions. Activity monitoring using activPAL devices is a validated method for quantifying physical activity in geriatric inpatients, except for measures of step count due to low gait speed. ${ }^{15}$ We derived information regarding the duration of upright and sedentary events from the manufacturer's comma-separated values (CSV) file using software V.7.3.32 (activPAL, PAL Technologies) and a custom MATLAB (MATLAB V.7.1. The MathWorks, Natick, Massachusetts, USA, 2005) program to export an Excel spreadsheet (Office Excel V.11.0, Windows XP Professional, Microsoft, 2003) with outcome values for all patients. The minimum length of an upright event for the sample was $10 \mathrm{~s}$. We used the activPAL Events file to determine the quantity and distribution of upright and sedentary events. We used upright time (minutes per 24 hours) and the total number of sit-to-stand transitions as measures of upright activity.

\section{ActiGraph outcomes}

The ActiGraph is a triaxial accelerometer usually worn on the wrist or the hip. ${ }^{19}{ }^{20}$ Using the ActiLife software (V.6.13.3), we filtered and accumulated the raw accelerometer signals into $1 \mathrm{~s}$ non-overlapping epochs and exported them to CSV files. We defined a threshold of 0.5 (units: ln $\left(\right.$ counts s $\left.\left.{ }^{-1}\right)\right)$ to separate static from dynamic behaviour of the wrist. We used the total number of counts above the threshold as an outcome measure of total wrist activity.

\section{Synchronised outcome}

We synchronised both devices using their respective timestamps and exported the synchronised data from both devices into CSV files. Based on time spent in a sedentary position, according to activPAL data, we generated a new variable indicating WAS. WAS describes the percentage of total sedentary time with wrist activity above the previously mentioned threshold.

\section{Baseline characteristics}

We collected demographic information on age, sex and nursing home stays from the patients' hospital records. We used the Short Physical Performance Battery ${ }^{21}$ (SPPB, $0-12,12$ is the best score), completed as early as possible during the hospital stay, as a measure of physical function. An SPPB score below 10 predicts all-cause mortality. ${ }^{22}$ We retrospectively completed the Global Deterioration Scale $^{23}$ as a measure of prehospital cognitive impairment (1-7, an increasing score indicates worse cognitive function) and the Barthel Index ${ }^{24}$ as a measure of prehospital personal activities of daily living ( $\mathrm{p}-\mathrm{ADL}$ ) function $(0-20$, an increasing score indicates better $\mathrm{p}$-ADL function). We calculated the Acute Physiology and Chronic
Health Evaluation II-score ${ }^{25}(0-71$, an increasing score indicates higher morbidity) and the Cumulative Illness Rating Scale ${ }^{26}(0-56$, a higher score indicates more serious chronic disease) as measures of morbidity and comorbidity.

\section{Statistical analysis}

We present descriptive statistics as means, $\mathrm{SD}$ and ranges for continuous and ordinal variables and as percentages for dichotomous variables. We checked data for normality by visual inspection of $Q-Q$ plots and compared subgroups using analysis of variance (ANOVA) with Scheffé correction for normally distributed variables and Kruskal-Wallis test/Mann Whitney U test with Dunn correction for non-normally distributed variables. We considered two-sided $p<0.05$ as significant. We used SPSS V.24 for all statistical analyses and report the results according to the Strengthening the Reporting of Observational Studies in Epidemiology cross-sectional reporting guidelines.

\section{Patients and public involvement}

Neither patients nor public were directly involved in the development of the research questions, study design, outcome measures, recruitment and conduct of the study. A summary of the main results will be communicated to the study participants on request.

\section{RESULTS}

We enrolled 311 patients, of whom 103 (33.1\%) had delirium. The final analysis included 60 patients with complete data from both devices for a 24-hour period with ongoing, motor subtyped, delirium. Among these, 15 had hyperactive, 20 hypoactive, 17 mixed and eight no-subtype delirium. Among those without complete activity monitoring, 10 had delirium only prior to arrival and could not be subtyped, 12 had hyperactive delirium, 10 had hypoactive delirium, 7 had mixed delirium and 4 had no-subtype delirium. Figure 1 shows the flow of patients. As shown in table 1 , the 60 patients had a mean age of 86.7 years (SD 5.2) and a mean SPPB score of 2.7 (SD 3.1). Table 2 shows activity monitoring data for all groups and $\mathrm{p}$ values for all pairwise comparisons. Figure 2 shows box plots illustrating different aspects of motor activity across motor subtypes.

\section{Upright activity}

Upright time and transitions were not normally distributed due to low levels of upright activity. The Kruskal-Wallis test showed significant differences between the groups for both upright time $(\mathrm{p}=0.015)$ and transitions $(\mathrm{p}=0.005)$. There were no significant differences between the hyperactive, hypoactive and mixed groups. We found, however, a significantly higher amount of upright time in the no-subtype group than in the hypoactive group (mean $119.3 \mathrm{~min}$ vs $37.8 \mathrm{~min}, \mathrm{p}=0.042$ ) and a significantly higher number of transitions in the no-subtype group than in both the hypoactive and mixed groups (mean 

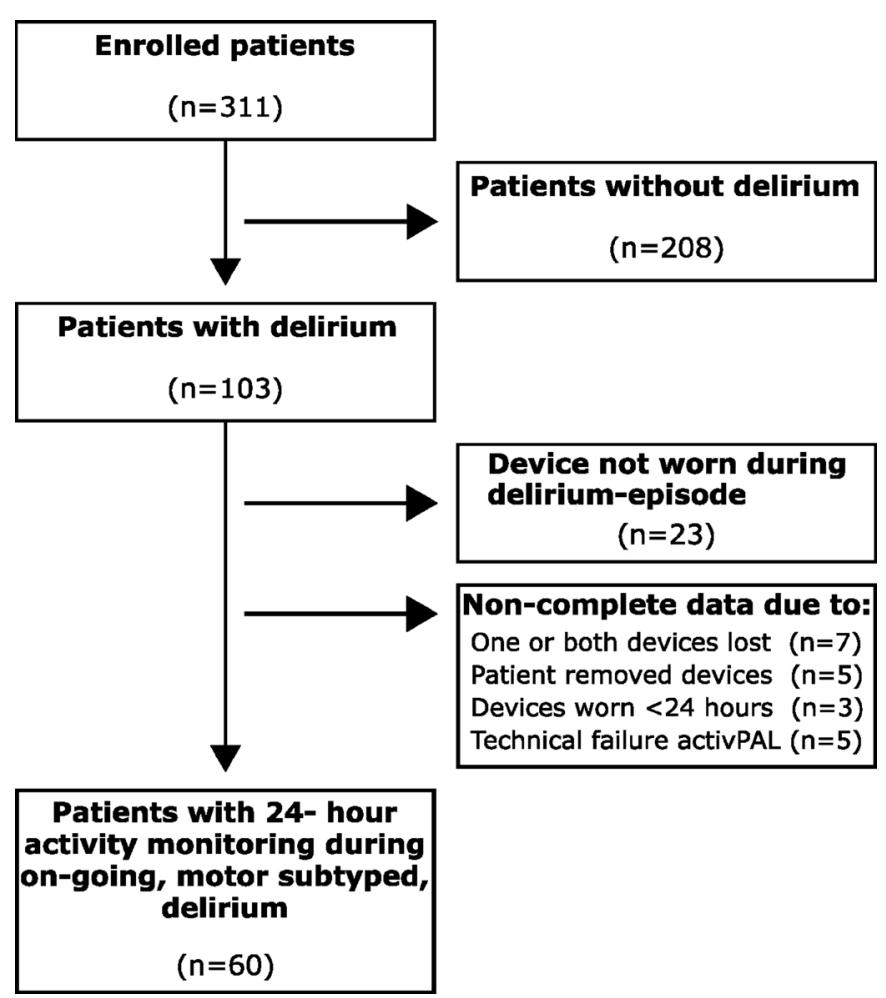

Figure 1 Flow chart summarising reasons why patients did not complete 24 hour activity monitoring.

no-subtype 54.3 vs hypoactive $17.4, \mathrm{p}=0.005$, and mixed $17.5, \mathrm{p}=0.013)$.

Total wrist activity

ANOVA analysis showed significant overall group differences for a total number of counts $(p=0.004)$. We found a significant difference between the hyperactive and the hypoactive group, with a significantly higher number of counts $\left(1238 \times 10^{4}\right.$ vs $\left.586 \times 10^{4}, \mathrm{p}=0.009\right)$ in the hyperactive group.

\section{Wrist activity in sedentary position}

ANOVA analysis showed significant overall group differences for WAS $(p=0.011)$. Comparing the hyperactive and the hypoactive group, we found a significantly higher amount of WAS (20\% vs $11 \%, \mathrm{p}=0.032$ ) in the hyperactive group. Comparing the mixed group and the hypoactive group, we found a significantly higher amount of WAS ( $19 \%$ vs $11 \%, \mathrm{p}=0.049)$ in the mixed group.

\section{DISCUSSION}

In this cross-sectional study on hospitalised geriatric patients with delirium, we found a low level of upright activity with no significant differences between the hyperactive, hypoactive and mixed groups. However, the no-subtype group had significantly more upright time than the hypoactive group and a significantly higher number of transitions than both the hypoactive and the mixed groups. In addition, we found significant differences in WAS between the hyperactive, hypoactive and the mixed groups, with higher amounts of WAS in the hyperactive and mixed groups than in the hypoactive group.

To our knowledge, this is the first study to simultaneously measure motor activity as both upright activity and wrist activity in geriatric patients with delirium motor subtyped by use of the DMSS, the only validated tool for motor subtyping. Godfrey conducted the only previous study analysing motor activity across DMSS-defined motor subtypes, but reported

Table 1 Baseline characteristics for patients with hyperactive, hypoactive, mixed and no-subtype delirium

\begin{tabular}{|c|c|c|c|c|c|}
\hline & $\begin{array}{l}\text { Hyperactive } \\
(n=15)\end{array}$ & $\begin{array}{l}\text { Hypoactive } \\
(n=20)\end{array}$ & $\begin{array}{l}\text { Mixed } \\
(n=17)\end{array}$ & $\begin{array}{l}\text { No-subtype } \\
(n=8)\end{array}$ & \\
\hline & \multicolumn{4}{|c|}{ Mean (SD) } & P value* $^{*}$ \\
\hline Age (years) & $86.3(6.3)$ & $85.5(4.4)$ & $88.7(4.7)$ & $86.1(5.8)$ & 0.28 \\
\hline p-ADL Function Barthel Index score (0-20) & $16.3(3.3)$ & $14.2(4.6)$ & $15.8(2.8)$ & $16.5(4.1)$ & 0.29 \\
\hline Cognitive Function GDS score (1-7) & $4.5(1.5)$ & $4.1(1.1)$ & $4.0(1.4)$ & $3.8(1.0)$ & 0.58 \\
\hline Physical Function SPPB score (0-12) & $4.2(3.8)$ & $1.2(1.5)$ & $2.1(2.9)$ & $5.1(3.0)$ & 0.003 \\
\hline Drugs on admittance (no) & $6.1(4.0)$ & $6.8(2.8)$ & $6.9(3.5)$ & $5.5(2.3)$ & 0.73 \\
\hline Acute IIIness APACHE II score (0-71) & $8.5(1.9)$ & $10.0(3.6)$ & $9.5(2.5)$ & $9.6(2.4)$ & 0.49 \\
\hline Comorbidity CIRS score (0-56) & $14.6(4.6)$ & $16.0(4.5)$ & $14.9(4.5)$ & $14.8(4.6)$ & 0.81 \\
\hline \multirow[t]{2}{*}{ Body mass index (kg/m²) } & $24.8(2.3)$ & $23.6(3.8)$ & $23.3(3.9)$ & $21.6(4.5)$ & 0.28 \\
\hline & \multicolumn{4}{|c|}{ No (\%) } & \\
\hline Female (\%) & $8(53)$ & $8(40)$ & $10(59)$ & $5(63)$ & 0.61 \\
\hline Living at home (\%) & $12(80)$ & $18(90)$ & 17 (100) & $8(100)$ & 0.17 \\
\hline Dementia† (\%) & $10(67)$ & $15(75)$ & $13(77)$ & $5(63)$ & 0.86 \\
\hline
\end{tabular}

${ }^{*} \mathrm{P}$ values are calculated using one-way analysis of variance for continuous variables and Pearson's $\chi^{2}$ test for categorical variables. †Global Deterioration Scale $\geq 4$.

CIRS, Cumulative IIIness Rating Scale; GDS, Global Deterioration Scale; p-ADL, personal activities of daily living; SPPB, Short Physical Performance Battery; APACHE II, Acute Physiology and Chronic Health Evaluation II. 
Table 2 Motor activity during 24 hours for the entire group and the four delirium motor subtypes, and pairwise comparisons of motor activity between delirium motor subtypes

Motor activity, mean (SD)

\section{Upright activity}

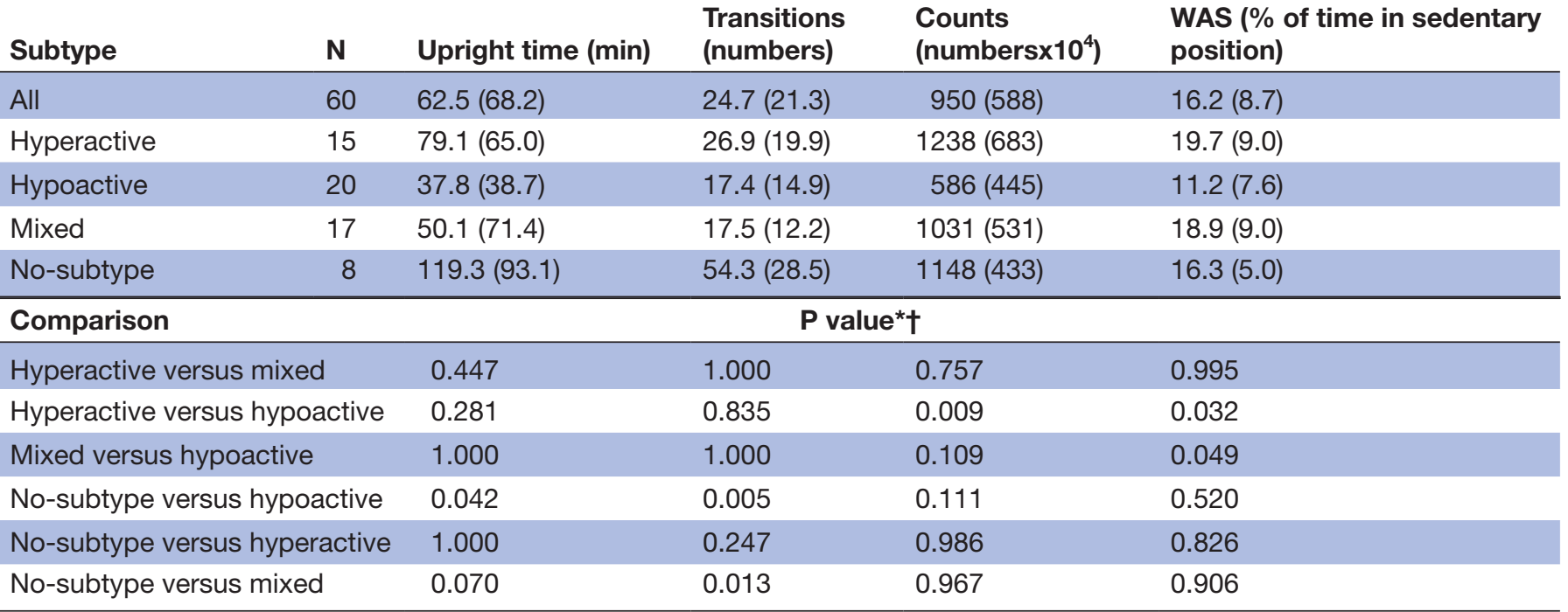

*Pairwise comparisons for upright activity are Dunn-corrected.

†Pairwise comparisons for wrist activity are Scheffé-corrected.

only upright activity. ${ }^{14}$ Patients included were substantially younger than our sample (mean age 70.7 years vs 86.7 years) and the overall finding was significant differences between the hyperactive and the hypoactive groups with the mixed group between the other groups. We could not reproduce these findings, probably because our patients
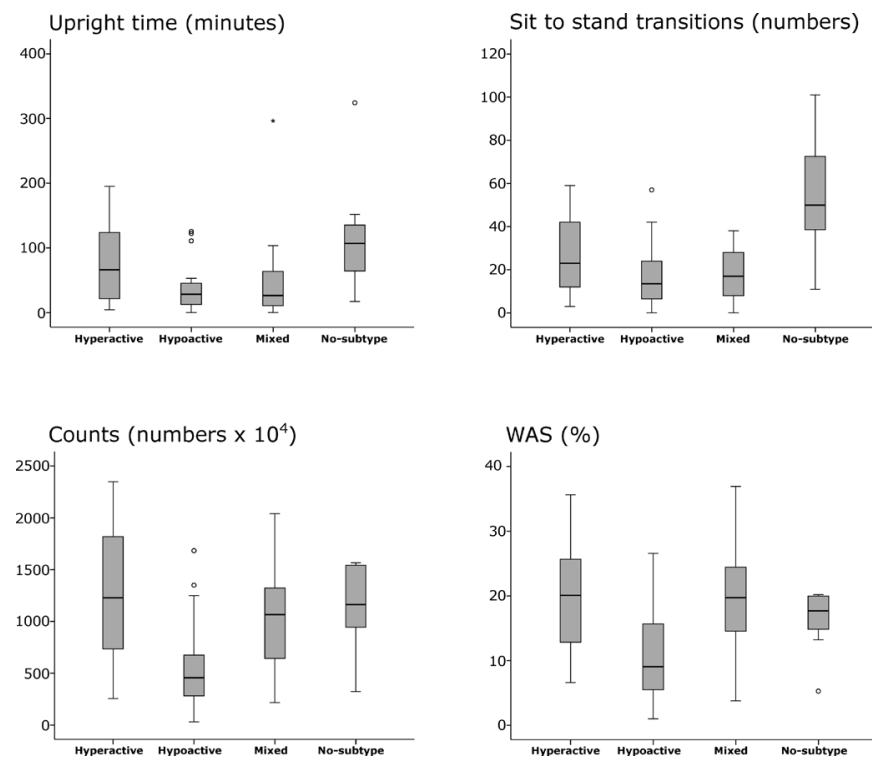

Figure 2 Box plots with delirium motor subtypes on the $\mathrm{x}$-axes and time in upright position, sit-to-stand transitions, counts and wrist activity in sedentary position (WAS) in per cent of the total time in sedentary position on the y-axes. The horizontal line in each box is the median, and the bottom and top of the boxes are the quartiles. are frail with heavily impaired gait function illustrated by a mean SPPB score of 2.7, and on a group level are incapable of expressing hyperactivity through increased amounts of upright activity. For total wrist activity, however, our results comply with Godfrey's with a significant difference between the hyperactive and the hypoactive groups and the mixed group between these. In our material, WAS separates the hypoactive group from both the hyperactive and mixed groups, which complies with the DMSS that states that both the hyperactive and the mixed groups have some sort of hyperactivity. In sum, the results from wrist actigraphy illustrate that also geriatric patients with delirium have motor disturbances that apply with the DMSS and can be captured by use of devices measuring aspects of motor activity other than upright time and that wrist activity, especially during sedentary behaviour, is a promising motor activity measure in frail, geriatric patients since it is independent of the patient's gait function.

Figure 2 illustrates a large variability within all groups for all measures of motor activity. This probably reflects that factors other than motor subtype also influence motor activity in geriatric patients with delirium, such as acute disease, frailty, brain pathology like Parkinson's disease and vascular dementia, lower limb function and sequelae after strokes and amputations. We identified discharge diagnoses with the potential of influencing motor activity in a negative way like strokes, fractures and difficulties to walk due to frailty or subcortical brain pathology in 33 out of 60 patients. In our sample, a low level of physical function is illustrated by low SPPB score in all groups, illustrating that geriatric patients with delirium are hardly able to get out 
of bed and walk. According to SPPB score, the hyperactive and the no-subtype groups seem to have a better physical function, but this might reflect the impact of the motor subtype on the SPPB performance rather than patients' physical function at baseline. When it comes to activity monitoring data, the patients with hyperactive delirium had low levels of upright activity, with a mean value of $79 \mathrm{~min}$ of upright time and 27 transitions per 24 hours. This illustrates that geriatric patients with delirium, and even those with hyperactive delirium, spend a majority of time in a sedentary position. Thus, clinicians cannot rely on wandering and changing of posture when looking for delirium. Our results indicate that patients with both hyperactive and mixed delirium express hyperactivity through wrist activity, and that clinicians should evaluate signs of restlessness in bed or in a chair rather than judging upright activity when looking for delirium.

This is the first study to report results of an accelerometer-based motor activity analysis in the no-subtype group. Some speculate that the no-subtype group represents less serious, questionable or resolving delirium. ${ }^{13} 27$ In our study, the main finding for this group was a higher amount of upright activity than both the hypoactive and mixed groups. The no-subtype group spent $119.3 \mathrm{~min}$ out of 24 hours in an upright position, which is almost identical to the $117.1 \mathrm{~min}$ of a general casemix of hospitalized geriatric patients that we have recently reported from our ward. ${ }^{16}$ This might indicate that the three motor subtypes hyperactive, hypoactive and mixed delirium represent fundamental motor disturbances eventually resulting in reduced motor activity, whereas the no-subtype represents a milder delirium not reaching the threshold for developing motor disturbances and that patients with no-subtype delirium thereby have a higher level of motor activity, more similar to patients without delirium. Thus, the important differences in motor activity in patients with delirium may be between the majority with motor symptoms and the minority without motor symptoms. This view is supported by a study finding that 38 patients with unsubtyped delirium had less wrist activity than 32 patients without delirium during the first 24 hours after cardiac surgery. ${ }^{28}$ In sum, the results from studies using activity monitoring in patients with delirium indicate that delirium, in general, is associated with less, and not more motor activity and also raises the question if the term 'hyperactive delirium' is misleading.

\section{Strengths and limitations}

The major strengths of this study are the simultaneous use of two accelerometer-based devices to measure both upright activity and wrist activity simultaneously and the use of DMSS for motor subtyping. This is also the first study to include the no-subtype group in analyses of motor activity. Our patients are old and frail. This is a strength since such patients frequently have delirium and were not recruited in previous research on delirium and activity monitoring, but also a limitation since our results are not necessarily applicable to patients with delirium in other settings. The sample of 60 patients is large compared with the only previous study in the field, although the small number of patients in each subgroup is the major limitation, creating a risk of type II error and preventing firm conclusions. There is also a possibility that patients with the most intense delirium were not included or did not complete activity monitoring, introducing a possible inclusion bias influencing the results. A potential bias is that patients with hyperactive delirium were slightly over-represented among those who did not complete 24 hours activity monitoring. Another important limitation is the cross-sectional design. A recent publication indicates that a substantial number of patients with delirium fluctuate between subtypes,${ }^{27}$ but we believe this has limited impact on our results since the activity monitoring was done in a limited time frame centred on the time of diagnosing and subtyping.

\section{CONCLUSIONS}

In this sample of frail, geriatric patients with delirium, we found a low level of upright activity with no differences between the hyperactive, hypoactive and mixed groups for neither upright time nor transitions. However, we found differences across these groups in both total wrist activity and WAS, indicating that there are true differences in motor activity across DMSS-defined motor subtypes also in geriatric patients with delirium. Our results indicate that restlessness while in a sedentary position is a more reliable clinical feature than wandering and changing of posture when looking for delirium in geriatric patients. Further research should address how motor features can improve the diagnostic workup of delirium in general and explore possible therapeutic consequences for the different delirium motor subtypes.

\section{Author affiliations}

${ }^{1}$ Department of Neuromedicine and Movement Science, Faculty of Medicine and Health Sciences, Norwegian University of Science and Technology, Trondheim, Norway

${ }^{2}$ Department of Geriatrics, St. Olavs Hospital, Trondheim University Hospital, Trondheim, Norway

${ }^{3}$ Regional Centre for Child and Youth Mental Health and Child Welfare, Department of Mental Health, Faculty of Medicine and Health Sciences, NTNU, Trondheim, Norway

${ }^{4}$ Oslo Delirium Research Group, Department of Geriatric Medicine, University of Oslo, Oslo, Norway

${ }^{5}$ Institute of Clinical Medicine, University of Oslo, Oslo, Norway

Acknowledgements Warm thanks to the staff at the geriatric ward for the help with inclusion and data collection. A special thanks to Helga Reklev for help with the activity monitors. Thanks to Phillipp Anders for help with creating figures. We are also grateful to participating patients and their supportive caregivers.

Contributors SE did the initial drafting of the article, had the main responsibility for data collection and for diagnosing and subtyping of delirium. AKB processed and analysed the activity monitoring data. OS is the project manager and designed the study. He also participated in diagnosing and subtyping of delirium. SL had the main responsibility for the statistical analyses. IS participated in designing and planning the study with particular responsibility in data collection at the geriatric ward. TBW participated in designing and planning the study with particular responsibility in the diagnostic workup of delirium and subtyping. KT participated in designing and planning the study, with a particular responsibility in planning data collection with 
the accelerometer-based devices. All authors have critically read and approved the final manuscript.

Funding The Liaison Committee for education, research and innovation in Central Norway funded the project.

Disclaimer We confirm that the funding body had no role in designing the study, in the collection, analysis or interpretation of data or in writing/submitting of the manuscript.

Competing interests AKB worked at NTNU, Department of Neuromedicine and Movement Sciences, when completing his contribution to this article. After finishing his contribution, but before the article was submitted, he started working at Roche Pharmaceutical Research and Early Development (pREDi), Roche Innovation Center Basel, F.Hoffmann-La Roche Ltt, 124 Grenzacherstrasse, Basel, CH 4070, Switzerland.

Patient consent for publication Not required.

Ethics approval The Regional Committee for Medical and Health Research Ethics of Mid-Norway approved the study (REK Central 2015/474).

Provenance and peer review Not commissioned; externally peer reviewed.

Data sharing statement Datasets from this study are not available since we do not have the consent to share the data neither from The Regional Committee for Medical and Health Research Ethics nor from the patients.

Open access This is an open access article distributed in accordance with the Creative Commons Attribution Non Commercial (CC BY-NC 4.0) license, which permits others to distribute, remix, adapt, build upon this work non-commercially, and license their derivative works on different terms, provided the original work is properly cited, appropriate credit is given, any changes made indicated, and the use is non-commercial. See: http://creativecommons.org/licenses/by-nc/4.0/.

\section{REFERENCES}

1. Inouye SK, Westendorp RG, Saczynski JS. Delirium in elderly people. Lancet 2014;383:911-22.

2. Witlox J, Eurelings LS, de Jonghe JF, et al. Delirium in elderly patients and the risk of postdischarge mortality, institutionalization, and dementia: a meta-analysis. JAMA 2010;304:443-51.

3. American Psychiatric Association. DSM-5 classification. Washington DC: American Psychiatric Publishing, 2013.

4. Marcantonio ER. Delirium in Hospitalized Older Adults. N Engl J Med 2017;377:1456-66.

5. Liptzin B, Levkoff SE. An empirical study of delirium subtypes. Br J Psychiatry 1992;161:843-5.

6. Bellelli G, Speciale S, Barisione E, et al. Delirium subtypes and 1-year mortality among elderly patients discharged from a post-acute rehabilitation facility. J Gerontol A Biol Sci Med Sci 2007;62:1182-3.

7. Kiely DK, Jones RN, Bergmann MA, et al. Association between psychomotor activity delirium subtypes and mortality among newly admitted post-acute facility patients. J Gerontol A Biol Sci Med Sci 2007;62:174-9.

8. Meagher DJ, Leonard M, Donnelly S, et al. A longitudinal study of motor subtypes in delirium: relationship with other phenomenology, etiology, medication exposure and prognosis. J Psychosom Res 2011;71:395-403.
9. Marcantonio E, Ta T, Duthie E, et al. Delirium severity and psychomotor types: their relationship with outcomes after hip fracture repair. J Am Geriatr Soc 2002;50:850-7.

10. Meagher DJ, Moran M, Raju B, et al. Motor symptoms in 100 patients with delirium versus control subjects: comparison of subtyping methods. Psychosomatics 2008;49:300-8.

11. Gupta N, de Jonghe J, Schieveld J, et al. Delirium phenomenology: what can we learn from the symptoms of delirium? J Psychosom Res 2008;65:215-22.

12. Meagher D, Moran M, Raju B, et al. A new data-based motor subtype schema for delirium. J Neuropsychiatry Clin Neurosci 2008;20:185-93.

13. Meagher DJ, Leonard M, Donnelly S, et al. A longitudinal study of motor subtypes in delirium: frequency and stability during episodes. $J$ Psychosom Res 2012;72:236-41.

14. Godfrey A, Leonard M, Donnelly S, et al. Validating a new clinical subtyping scheme for delirium with electronic motion analysis. Psychiatry Res 2010;178:186-90.

15. Taraldsen K, Askim T, Sletvold O, et al. Evaluation of a body-worn sensor system to measure physical activity in older people with impaired function. Phys Ther 2011;91:277-85.

16. Evensen S, Sletvold O, Lydersen S, et al. Physical activity among hospitalized older adults - an observational study. BMC Geriatr 2017; $17: 110$

17. Ellis G, Gardner M, Tsiachristas A, et al. Comprehensive geriatric assessment for older adults admitted to hospital. Cochrane Database Syst Rev 2017;9:CD006211.

18. Saczynski JS, Kosar CM, Xu G, et al. A tale of two methods: chart and interview methods for identifying delirium. J Am Geriatr Soc 2014;62:518-24.

19. Martin JL, Hakim AD. Wrist actigraphy. Chest 2011;139:1514-27.

20. Jefferis BJ, Parsons TJ, Sartini C, et al. Objectively measured physical activity, sedentary behaviour and all-cause mortality in older men: does volume of activity matter more than pattern of accumulation? Br J Sports Med 2018:bjsports-2017-098733.

21. Guralnik JM, Simonsick EM, Ferrucci L, et al. A short physical performance battery assessing lower extremity function: association with self-reported disability and prediction of mortality and nursing home admission. J Gerontol 1994;49:M85-94.

22. Pavasini R, Guralnik J, Brown JC, et al. Short physical performance battery and all-cause mortality: systematic review and meta-analysis. BMC Med 2016;14:215.

23. Reisberg B, Ferris SH, de Leon MJ, et al. The global deterioration scale for assessment of primary degenerative dementia. Am $J$ Psychiatry 1982;139:1136-9.

24. Mahoney FI, Barthel DW. Functional evaluation: the barthel index. Md State Med J 1965;14:61-5.

25. Knaus WA, Draper EA, Wagner DP, et al. APACHE II: a severity of disease classification system. Crit Care Med 1985;13:818-29.

26. Salvi F, Miller MD, Grilli A, et al. A manual of guidelines to score the modified cumulative illness rating scale and its validation in acute hospitalized elderly patients. J Am Geriatr Soc 2008;56:1926-31.

27. Scholtens RM, van Munster BC, Adamis D, et al. Variability of delirium motor subtype scale-defined delirium motor subtypes in elderly adults with hip fracture: a longitudinal study. J Am Geriatr Soc 2017;65:e45-e50.

28. Osse RJ, Tulen JH, Hengeveld MW, et al. Screening methods for delirium: early diagnosis by means of objective quantification of motor activity patterns using wrist-actigraphy. Interact Cardiovasc Thorac Surg 2009;8:344-8. discussion 348. 